\title{
Autoreactive B-cells phenotype analysis in pemphigus patients before and after anti-CD20 treatment
}

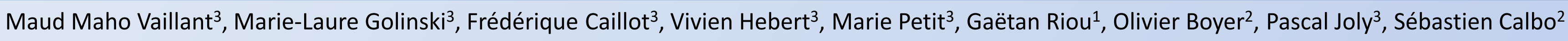
1. INSERM Unit 1234, Rouen, France.

2. INSERM Unit 1234 and Normandy University, Rouen, France.

3. Department of Dermatology, Rouen University Hospital and INSERM unit 1234, Rouen, France.

\section{AIM OF STUDY}

Pemphigus is a B-cell-mediated autoimmune disease affecting skin and mucous membranes. Pathogenic autoantibodies are directed against desmogleins (DSG-1/DSG-3), which are keratinocyte adhesion proteins. In a previous study, we have shown that B cell-depletion by Rituximab (RTX), an anti-CD20 antibody used as first line treatment induced a $89 \%$ rate of complete remission off therapy (CR) at month 24. Patients were followed up to month 36 (M36) after RTX treatment to understand the immunological mechanisms which mediate this long lasting CR. We aimed at characterizing the renewed B cells and DSG-specific B cell subpopulations in peripheral blood of pemphigus patients.

\section{METHODS}

Phenotype of B cells and DSG-1 or DSG-3 specific B cells were studied by flow cytometry using recombinant DSG HIS-tagged and an antipoly HIS antibody, in healthy controls $(H C ; n=14)$ and in pemphigus patients ( $P V ; n=17)$ at day 0 and M36 after first RTX treatment.

Gating strategy for analysis of B cells and DSG specific B cells subpopulation in peripheral blood after B cell enrichment

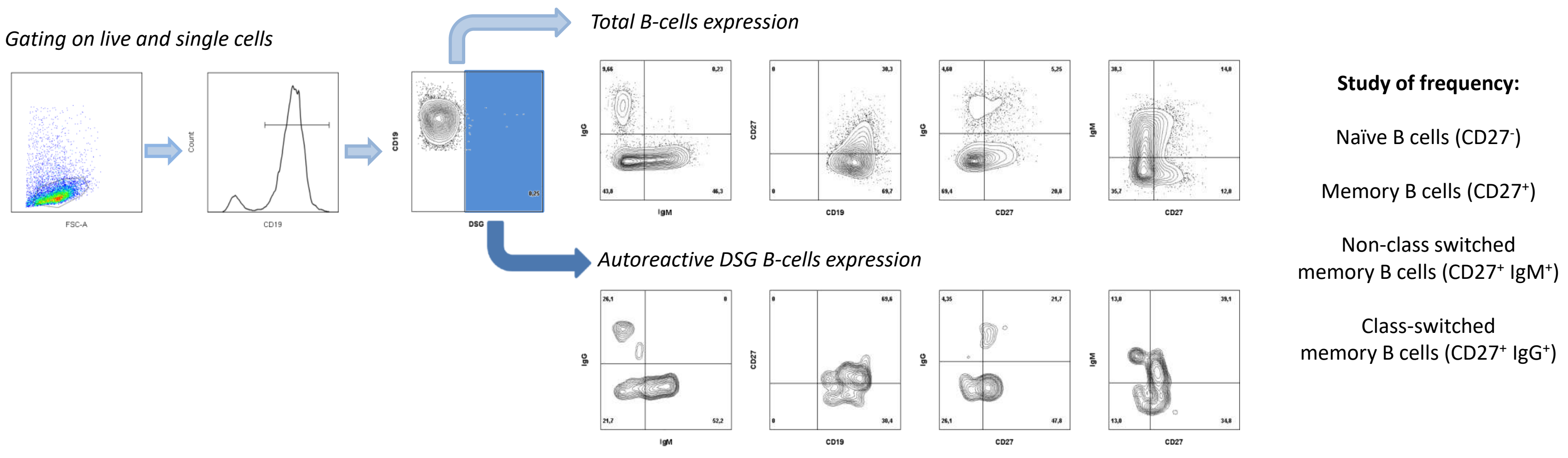

Frequency of DSG specific B cells

in PV patients at day 0 and in HD

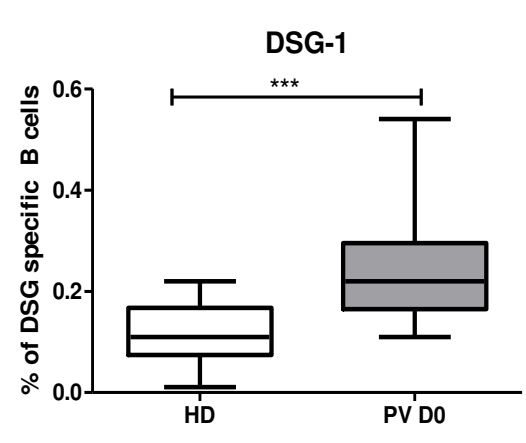

Mann Whitney test -
Bonferroni correction Bonferroni correctio
$(* * * p<0,001)$

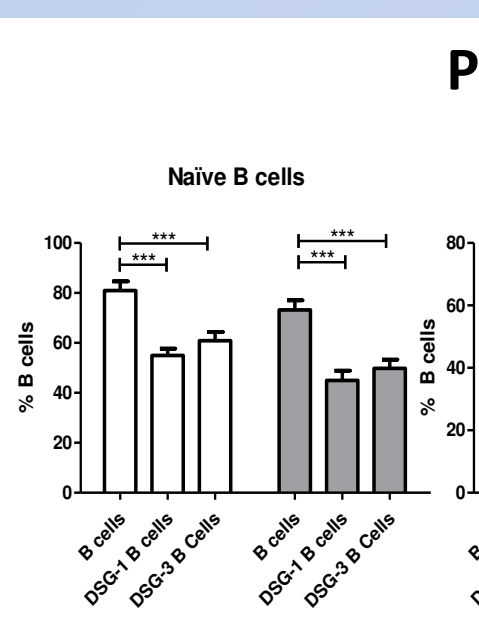

Phenotype of DSG specific B cells
Autoreactive DSG specific B cells were detected in PV patients and HD. Frequency of these cells was significantly higher in these newly diagnosed pemphigus than in $H D$.
Autoreactive B cells were enriched in memory B cells compare to total B cells population. PV patients had high frequency of class swiched IgG memory B cells in their autoreactive population.

Evolution of B cells and DSG specific B cells in PV in CR at M36 after first RTX treatment

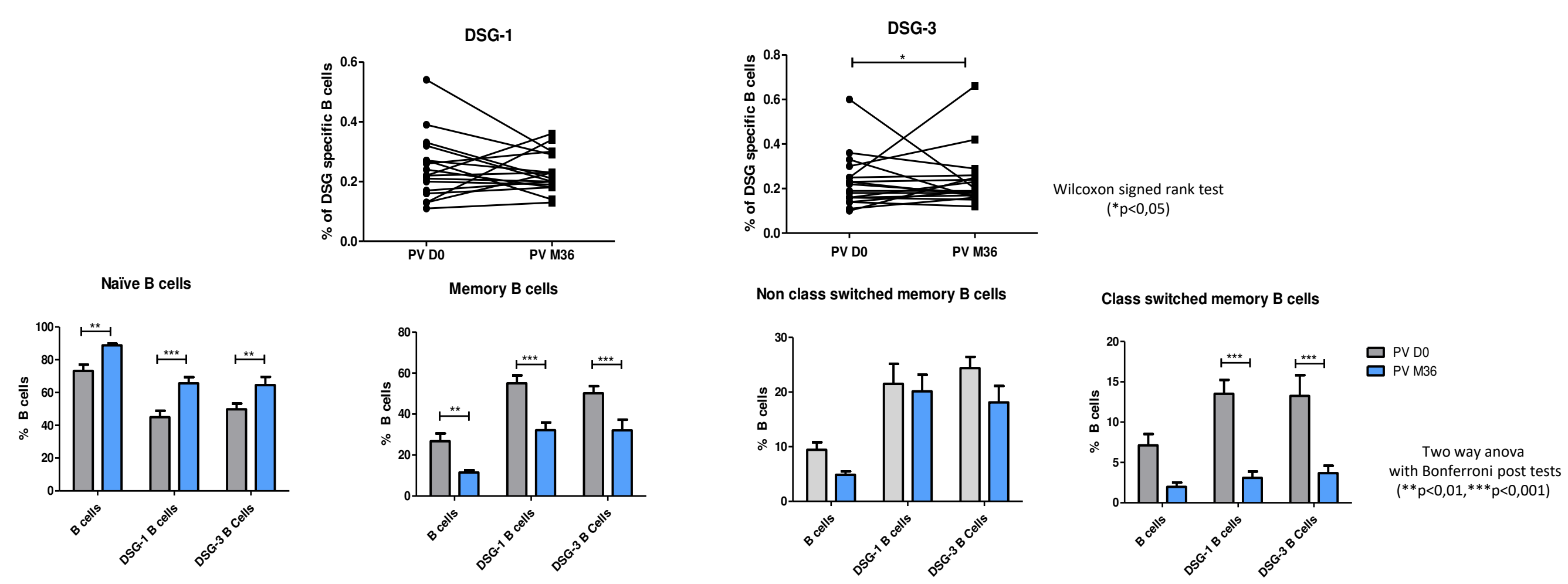

Balance between Naive and Memory B cells is modified after RTX.

Autoreactive DSG-specific B cells were still detected at M36 but their phenotypes were impacted by RTX.

The frequency of class switched memory was significantly decrease compared to baseline.

\section{CONCLUSION}

DSG-specific B cells are present in peripheral blood of PV patients even in patients in CR. DSG-specific class switched IgG memory B cells are enriched during acute phase and were markedly reduced after RTX. This population seems to play a major role in PV disease. 